\title{
Clinical Study \\ Risk of Endophthalmitis in Boston Type 1 Keratoprosthesis Combined with Vitrectomy and Silicone Oil Insertion
}

\author{
Mohamed Abou Shousha $\mathbb{D D}^{1,2}$ Taher Eleiwa $\mathbb{D}^{2,3}$ Allister Gibbons $\mathbb{D}^{2},{ }^{2}$ Christopher Smith, \\ Sean Edelstein, ${ }^{1}$ George Kontadakis $\left(\mathbb{D},{ }^{2}\right.$ Zachary Schmitz, ${ }^{1}$ Joshua Abernathy, \\ Ross Chod, ${ }^{1}$ Zachary Bodnar, ${ }^{1}$ Kelvin McDaniel, ${ }^{1}$ Rocio Bentivegna, ${ }^{1}$ and Levent Akduman ${ }^{1}$ \\ ${ }^{1}$ Saint Louis University Eye Institute, St Louis, MO, USA \\ ${ }^{2}$ Bascom Palmer Eye Institute, Miami, FL, USA \\ ${ }^{3}$ Department of Ophthalmology, Faculty of Medicine, Benha University, Benha, Egypt
}

Correspondence should be addressed to Mohamed Abou Shousha; mshousha@med.miami.edu

Received 6 December 2018; Revised 15 March 2019; Accepted 5 May 2019; Published 25 July 2019

Academic Editor: Wisam A. Shihadeh

Copyright (c) 2019 Mohamed Abou Shousha et al. This is an open access article distributed under the Creative Commons Attribution License, which permits unrestricted use, distribution, and reproduction in any medium, provided the original work is properly cited.

\begin{abstract}
Purpose. To identify the incidence of endophthalmitis and visual outcomes in eyes with Boston type 1 keratoprosthesis combined with pars plana vitrectomy and silicone oil insertion (KPro + PPV + SOI) as compared to eyes receiving Boston type 1 keratoprosthesis (KPro) alone. Patients and Methods. Retrospective chart review of 29 eyes of 27 patients with KPro having at least 12month follow-up. Thirteen of these eyes had hypotony and/or retinal detachment in addition to corneal pathology and thus received KPro + PPV + SOI. Polymyxin-trimethoprim with a quinolone was used as chronic topical antibiotic prophylaxis in both groups after the first postoperative month. Outcome measures recorded at the 1-, 3-, 6-, 12-, and 24-month follow-up visits included best-corrected visual acuity (BCVA) and rates of postoperative complications. Results. All the patients had completed 24month follow-up except one case in the KPro group who lost to follow-up after 12-month visit. In the KPro + PPV + SOI group, no eyes had developed endophthalmitis by the 24-month follow-up visit versus 5 eyes of 5 patients in the uncombined KPro group $(P=0.048)$. The 2 -year cumulative endophthalmitis incidence was $31.2 \%$ in the KPro group versus zero in the KPro + PPV + SOI group $(P=0.030)$. Four of these 5 eyes had vitreous taps with positive cultures; 2 were positive with Staphylococcus aureus, 1 with coagulase-negative staphylococci, and 1 with Streptococcus pneumoniae. Other complications included KPro extrusion (1 in each group), retinal detachment ( 2 in the KPro and 1 in the KPro + PPV + SOI group), newly developed glaucoma (2 in each group), and retroprosthetic membrane ( 9 in the KPro and 5 in the KPro + PPV + SOI group). The KPro group had better average preoperative BCVA compared to those of the KPro + PPV + SOI group $(-2.29 \pm 0.72$ LogMAR, versus $-2.95 \pm 0.30 \operatorname{LogMAR}$; $P=0.004)$. No statistically significant difference in BCVA was noted in subsequent follow-up visits. Conclusion. The addition of PPV and SOI to the KPro implantation in the eyes with corneal pathology, as well as hypotony and/or retinal detachment, is a safe and effective procedure for visual rehabilitation. Pars plana vitrectomy and silicone oil insertion may have a protective effect against the development of postoperative endophthalmitis in eyes receiving KPro.
\end{abstract}

\section{Introduction}

The Boston type I keratoprosthesis (Massachusetts Eye and Ear Infirmary, Boston, MA; KPro) is the most widely used prosthetic corneal transplant in the United States and the world [1]. KPro has gained popularity over the last decade. The number of KPro procedures has increased from fewer than 50 in 2002 to more than 1150 in 2009 [2]. The goal of using a KPro is to attempt to restore vision in patients who would otherwise have a very poor prognosis with penetrating keratoplasty. This subset of patients includes those with previous graft failures, limbal stem cell deficiency, cicatrizing diseases, and chemical injuries $[2,3]$.

Outcomes of KPro implantation have been encouraging. Nevertheless, long-term studies have shown a high rate of sight-threatening complications with a device retention rate 
of only $67 \%$ at 7 years, highly dependent on the KPro indication [4]. One catastrophic complication of KPro is infectious endophthalmitis. Long-term studies have shown a 7 -year cumulative endophthalmitis incidence up to $15.5 \%$ after Boston type 1 KPro $[4,5]$ versus a 5-year cumulative incidence of $1.3 \%$ for bleb-related endophthalmitis [6] and $6.3 \%$ of endophthalmitis after glaucoma drainage device insertion [7], with both sharing the ongoing risk of infection and worse visual outcomes compared to infectious endophthalmitis after penetrating keratoplasty and cataract surgery. The KPro is a device, with limited biointegration, that bridges a nonsterile ocular surface with a sterile anterior chamber and can lead to rapid invasion of pathogenic organisms through the space between the tissue and the prosthesis [8].

In this study, we report our observation that patients with KPro combined with pars plana vitrectomy and silicone oil insertion have a lower incidence of infectious endophthalmitis than those with KPro alone.

\section{Materials and Methods}

This was a retrospective chart review of patients who underwent KPro implantation and patients who underwent KPro implantation in combination with pars plana vitrectomy (PPV) and silicone oil insertion (SOI) in Saint Louis University Eye Institute (SLUEI) and Bascom Palmer Eye Institute from January 2011 until January 2018. This study was approved by Saint Louis University and the University of Miami Institutional Review Board. Indications for KPro transplantation are listed in Table 1. Eyes that had hypotony and/or retinal detachment in addition to corneal pathology received KPro implantation combined with PPV and SOI. Implantation of KPro in all cases was performed by previously published techniques [9]. Patients, who planned to undergo PPV and SOI, initially underwent an Eckhardt temporary keratoprosthesis implantation followed by PPV and SOI by a retina specialist and then replacement of Eckhardt keratoprosthesis with KPro; all procedures were performed in the same session.

Best-corrected distance visual acuity (BCVA), intraocular pressure, slit lamp examination, and complications were reviewed at the 1-, 3-, 6-, 12-, and 24-months postoperative follow-up visits. The start date of the follow-up was the date of KPro insertion, and the end date was the last date the patient was seen by the ophthalmologist, or KPro removed, or the patient lost all vision in the KPro eye. The patient should have at least 12-month follow-up to be included in the study. Endophthalmitis, visual outcomes, and other complications were compared between groups.

Statistical analysis with SPSS software version 20.0 (SPSS, Chicago, IL, USA) was performed to calculate descriptive statistics for all eyes. Rates of complications were compared between groups by means of chi-squared test. Average visual acuity and duration of follow-up were compared between both groups with two sample $t$-tests. Average visual acuity was compared at different follow-up visits for the endophthalmitis cases using repeated measures analysis of variance (ANOVA). The time-related cumulative incidence of endophthalmitis in each category was evaluated by means of Kaplan-Meier survival curves. Two-sided $p$ values less than 0.05 were considered statistically significant. Values are presented as means \pm standard deviation.

\section{Results}

The study included 29 eyes of 27 patients. Sixteen eyes underwent only KPro implantation (KPro group), and 13 eyes underwent KPro implantation combined with PPV and SOI (KPro + PPV + SOI group). The mean follow-up was 23 months (range 12-24 months) in the KPro group, versus 24 months in the KPro + PPV + SOI group $(P=0.377)$, with a $93.75 \%$ of KPro group and $100 \%$ of KPro + PPV + SOI group completing 24-month follow-up. Table 2 summarizes the different features of both groups. The early postoperative management (1 month) included the use of polymyxin B sulfate and trimethoprim (Polytrim, Allergan Inc., Irvine, CA) and ofloxacin $0.3 \%$ (Ocuflox, Allergan, Irvine, CA) or moxifloxacin $0.5 \%$ (Vigamox, Alcon Inc., Fort Worth, TX) in both groups. This was in addition to topical steroids in both groups; the KPro $+\mathrm{PPV}+\mathrm{SOI}$ group received dexamethasone and tobramycin (Tobradex; Alcon Labs, Fort Worth, TX), while the KPro group received prednisolone acetate 1\% (Pred Forte; Allergan, Irvine, CA). All drops were administered four times daily. Past the 1-month follow-up visit, all patients were kept chronically on one regimen, polymyxin B sulfate and trimethoprim (Polytrim, Allergan Inc., Irvine, CA) and ofloxacin 0.3\% (Ocuflox, Allergan, Irvine, CA) or moxifloxacin 0.5\% (Vigamox, Alcon Inc., Fort Worth, TX) administered twice daily. Vancomycin-fortified eye drops were not prescribed for prophylaxis due to availability and cost issues. Four patients in the KPro group and 6 patients in the KPro + PPV + SOI group did not use contact lenses chronically secondary to intolerance. There were no persistent epithelial defects or infectious keratitis of the corneal carrier tissue noted in any of the included cases.

During the 24-month follow-up period, no eyes in the $\mathrm{KPro}+\mathrm{PPV}+\mathrm{SOI}$ group developed endophthalmitis versus 5 eyes of 5 patients in the KPro group ( $P=0.048$, Fisher's exact test). Table 2 summarizes the characteristics of the 5 cases. Endophthalmitis occurred within a range of 210 months. All the cases were compliant with their topical antibiotic drops at the time of onset of endophthalmitis. The Kaplan-Meier analysis for the incidence of endophthalmitis in the KPro versus KPro + PPV + SOI groups is given in Figure 1. Among those managed with the KPro + PPV + SOI procedure, the incidence of endophthalmitis was zero at two-year follow-up, while the uncombined KPro group had a 2-year cumulative incidence \pm SE of endophthalmitis of $31.2 \% \pm 11.6 \% \quad(P=0.030)$. Patients who developed endophthalmitis received an intravitreal tap and injection of antibiotics. Vitreous aspirate cultures were positive in 4 cases: Staphylococcus aureus (2 cases), coagulase-negative staphylococci (1), and Streptococcus pneumoniae (1). There was no significant difference in the rate of endophthalmitis between patients who used contact lenses chronically versus those who did not. The KPro was not removed in the 5 cases, and they maintain potential vision till the last date of follow- 
TABLE 1: Indications for surgery.

\begin{tabular}{|c|c|c|c|}
\hline \multicolumn{2}{|c|}{ Boston type 1 keratoprosthesis group $(N=16)$} & \multicolumn{2}{|c|}{$\begin{array}{l}\text { Boston type } 1 \text { keratoprosthesis combined with vitrectomy and } \\
\text { silicone oil insertion group }(N=13)\end{array}$} \\
\hline Indication & Total & Indication & Total \\
\hline Multiple graft failures & 10 & Multiple graft failures and retinal detachment & 4 \\
\hline Aniridia and limbal stem cell deficiency & 3 & $\begin{array}{c}\text { Severe alkaline injury, graft failure, and retinal } \\
\text { detachment }\end{array}$ & 3 \\
\hline Scleroderma and multiple graft failure & 1 & Chronic uveitis with hypotony & 2 \\
\hline Herpetic keratitis and neurotrophic ulcer & 1 & Hypotony and graft failure & 2 \\
\hline Stevens-Johnson syndrome & 1 & $\begin{array}{c}\text { Herpetic keratitis, neurotrophic ulcer with retinal } \\
\text { detachment }\end{array}$ & 1 \\
\hline & & Aniridia, limbal stem cell deficiency, and hypotony & 1 \\
\hline
\end{tabular}

TABLE 2: Characteristics of the studied groups.

\begin{tabular}{|c|c|c|c|}
\hline & & Studie & roups \\
\hline & & KPro & $\mathrm{KPro}+\mathrm{PPV}+\mathrm{SOI}$ \\
\hline Age $($ mean $\pm S D)$ & & $61 \pm 18$ years & $56 \pm 25$ years \\
\hline Follow-up (mean; range) & & 23 (12-24) months & 24 months \\
\hline Number (\%) with follow-up to 6 months & & $16(100)$ & $13(100)$ \\
\hline Number (\%) with follow-up to 12 months & & $16(100)$ & $13(100)$ \\
\hline Number (\%) with follow-up to 24 months & & $15(93.75)$ & $13(100)$ \\
\hline Number (\%) with glaucoma drainage implant & & $1(7.69)$ & $9(56.25)$ \\
\hline Number (\%) wearing bandage contact lenses & & $12(75)$ & $7(65.5)$ \\
\hline Number (\%) using prophylactic topical antibiotic & Polytrim + ofloxacin $0.3 \%$ & $9(56)$ & $7(54)$ \\
\hline regimen & Polytrim + moxifloxacin $0.05 \%$ & $7(44)$ & $6(46)$ \\
\hline
\end{tabular}

up (Table 3). Only one case was lost to follow-up after the 12month visit. Figure 2 demonstrates the visual performance in endophthalmitis cases.

Other complications that occurred among both groups are seen in Figure 3. Each group had one KPro extrusion, as well as 2 newly developed glaucoma $(P=1.0)$. Retroprosthetic membranes were common among each group, 9 occurring in the KPro and 5 in the KPro + PPV + SOI group $(P=0.340)$. Two of the patients in the KPro versus 1 in the $\mathrm{KPro}+\mathrm{PPV}+\mathrm{SOI}$ group had retinal detachment $(P=1.0)$. Nine of the patients in the KPro-only group had a glaucoma drainage implant (GDI) versus only one in the combined group $(P=0.008)$. Two of those patients with the GDI developed endophthalmitis without evidence of GDI infection. There was no statistically significant difference between incidences of endophthalmitis in patients with or without GDI in our case series $(P=0.549)$. One of the patients in each group had a corneal melt that led to extrusion, yet it was noted that the retroprosthetic membrane was very dense and there was no leakage of the intraocular contents (Figure 4).

The KPro-only group had better average preoperative BCVA as compared to those of the KPro + PPV + SOI group (Figure 5). BCVA preoperatively in the KPro group was $-2.29 \pm 0.72 \operatorname{LogMAR}$ (Snellen equivalent of $1 / 200$ ) versus $-2.95 \pm 0.30$ LogMAR (Snellen equivalent of $\mathrm{HM}$ ) in the combined group ( $P=0.004, t$-test). After the surgery, BCVA was not statistically significant between each group.

\section{Discussion}

The results of this study suggest that pars plana vitrectomy and silicone oil placement could lower the rate of endophthalmitis in eyes with Boston type 1 keratoprosthesis. Boston type 1 keratoprosthesis provide the ability to restore vision in selected patients with corneal blindness when a corneal transplant is estimated to have a worse outcome. As with any keratoplasty, this is associated with an increased risk of endophthalmitis [10]. Between the years of 1999-2009, one large academic institution published a rate of endophthalmitis from all intraocular surgeries to be $0.065 \%$ and from cataract surgery to be $0.041 \%$ [11]. Bleb-related endophthalmitis is the second most frequent cause of postoperative endophthalmitis after acute and chronic postcataract surgery endophthalmitis [12]. Its incidence is reported to be between $0.2 \%$ and $1.3 \%,[13,14]$ and increases with the use of antiproliferative agents (up to $3 \%$ ) and with inferiorly placed blebs (up to 9.4\%) [15-18]. Both bleb and KPro-related endophthalmitis share the same ongoing risk of infection, virulent organisms crossing an altered barrier between the ocular surface and the aqueous, late onset endophthalmitis and poor visual outcome [19]. Taban et al. reported a rate of endophthalmitis of $0.382 \%$ for penetrating keratoplasty [20]. Rishi et al. reported an incidence of $9 \%$ over a ten-year follow-up period with Boston keratoprosthesis type 1 [21]. As discussed earlier, the rate of endophthalmitis in patients with KPro has been reported to be as high as $15.5 \%$ over a 7 -year followup period $[4,5]$. In this study, the overall incidence of endophthalmitis within the follow-up period was $17 \%$; however, it was higher in the uncombined KPro group. Grassi et al. reported that sterile vitritis after KPro can mimic infectious endophthalmitis with pain and external signs of inflammation [22]; however, the absence of overt 


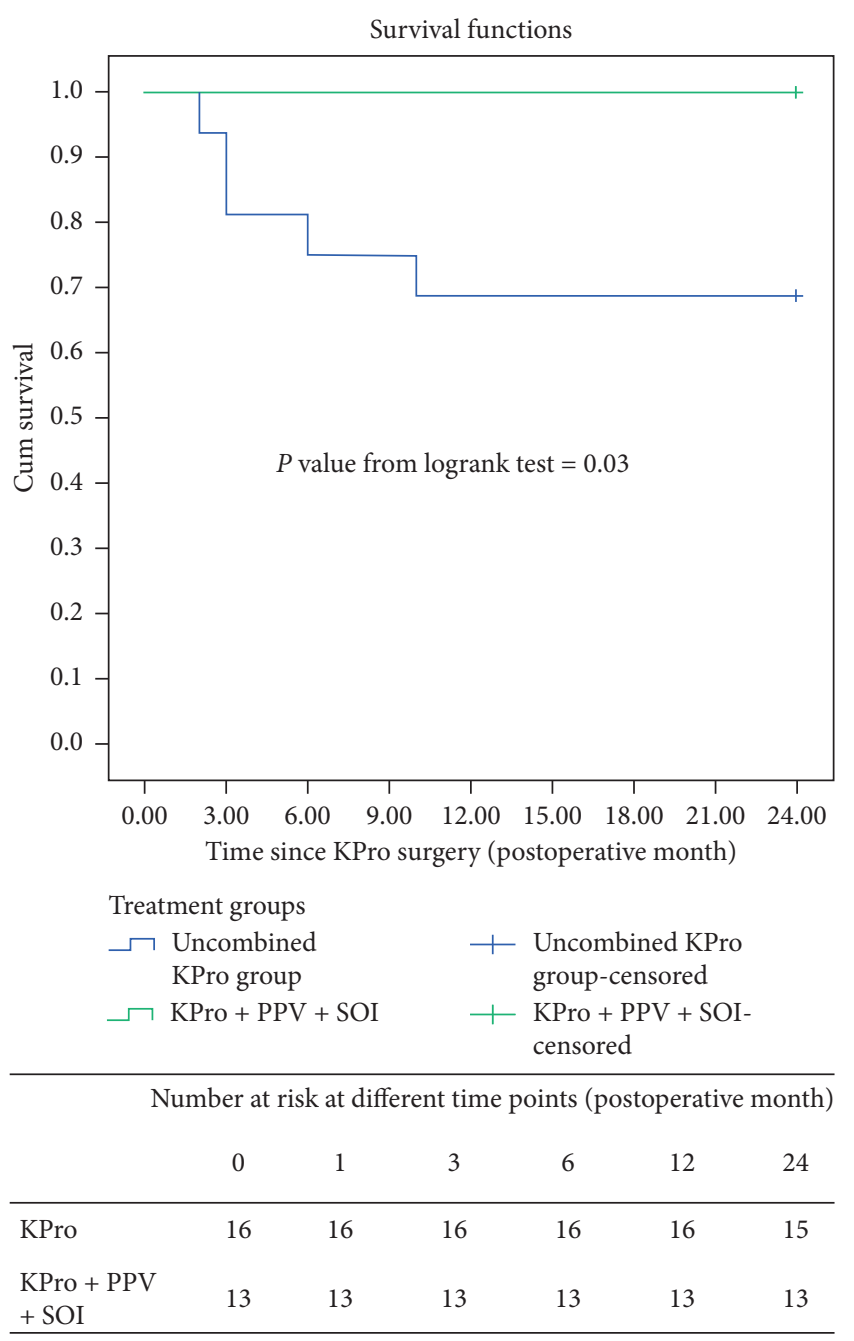

FIGURE 1: Kaplan-Meier graph of the incidence of endophthalmitis in the Boston type 1 keratoprosthesis (uncombined KPro) group versus combined KPro, pars plana vitrectomy, and silicone oil insertion group (KPro + PPV + SOI), with the number of subjects at risk at different time points listed underneath the figure. The 2-year cumulative (Cum) survival ratio was $68.8 \%$ in the KPro group, versus $100 \%$ in the KPro + PPV + SOI group $(P=0.03)$.

clinical signs does not rule out infection either [23]. The prognosis also appears to be worse when endophthalmitis occurs after KPro implantation, versus other surgeries [5]. Endophthalmitis isolates in our study were all Grampositive. Gram-positive bacteria were found predominant in endophthalmitis $[10,19,24-27]$. No susceptibility data were obtained in our study. However, according to the literature, Gentile et al. reported susceptibility to fluoroquinolones for Gram-positive isolates from a low of $65 \%$ for levofloxacin to a high of $78 \%$ for gatifloxacin, versus $99.7 \%$ to vancomycin during the time period from 1987 to 2011 [24]. Durand et al. reported a lower incidence of bacterial endophthalmitis (1\%) in KPro eyes with prophylactic topical vancomycin, given its excellent Gram-positive coverage [19]; however, it was not prescribed in this study for prophylaxis due to availability and cost issues.

In our study, the 2-year endophthalmitis rate was lower when KPro was combined with pars plana vitrectomy and intraocular silicone oil $(P=0.03)$. One possible mechanism is that silicone oil has antimicrobial properties. Özdamar et al. tested the antimicrobial properties by incubating silicone oil with several known endophthalmitis-associated microorganisms. Results showed a decrease in growth in all of the colonies incubated with silicone oil [28]. Another mechanism may be that silicone oil leads to the formation of a strong retroprosthetic membrane that acts as a mechanical barrier to pathogenic organisms. An image of this membrane can be seen in Figure 4 . While, we understand that silicone oil insertion has its own complications and that it is not recommended to place silicone oil for mere prophylaxis, we believe that our observation has the potential to direct future research in the development of new keratoprosthesis to avoid the unacceptable high rate of postoperative complications.

Silicone oil is used in order to facilitate intraocular tamponade. It is thought to prevent uveal edema and hypotony by maintaining long-term pressure in the eye. This avoids retinal and choroidal detachments from occurring [29]. In our cases, silicone oil placement was used to treat 


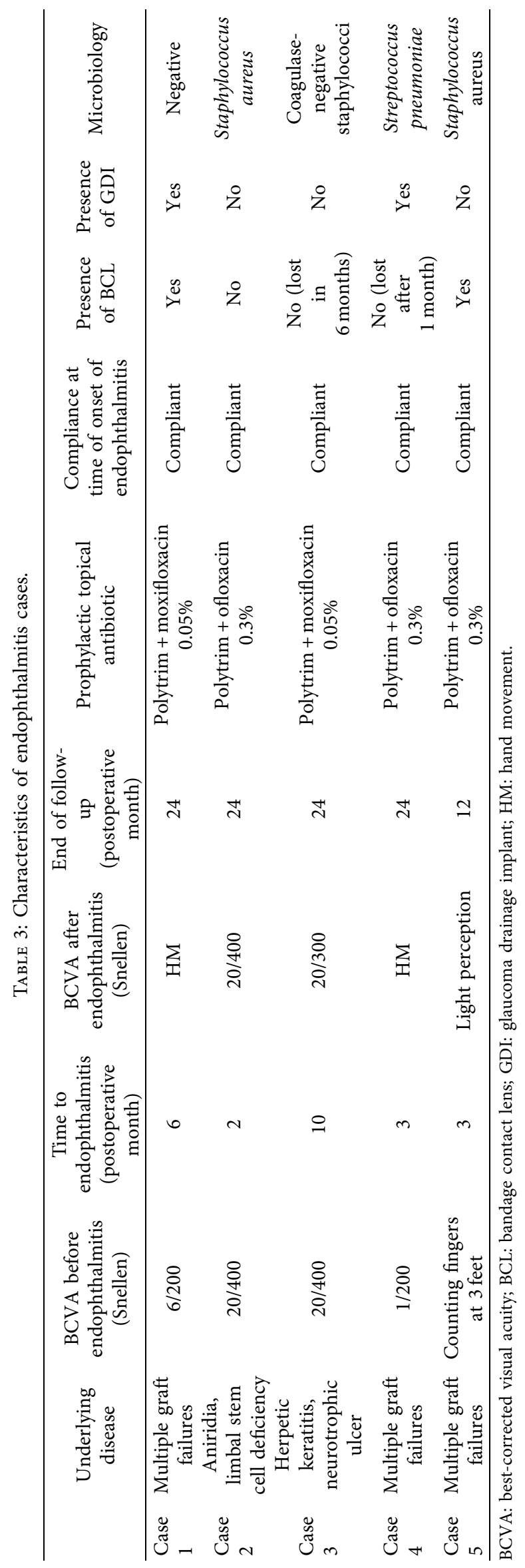




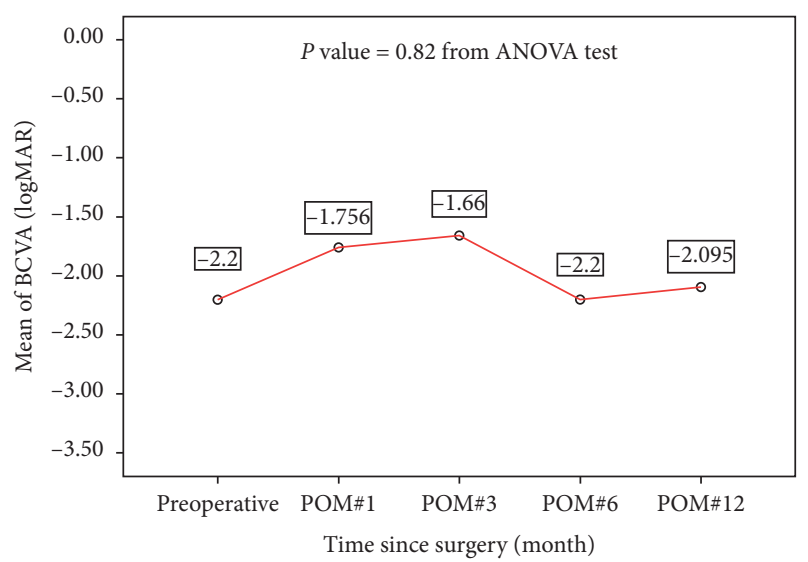

Figure 2: Graph illustrating visual performance in endophthalmitis cases at different follow-up visits. POM: postoperative month; ANOVA: analysis of variance.

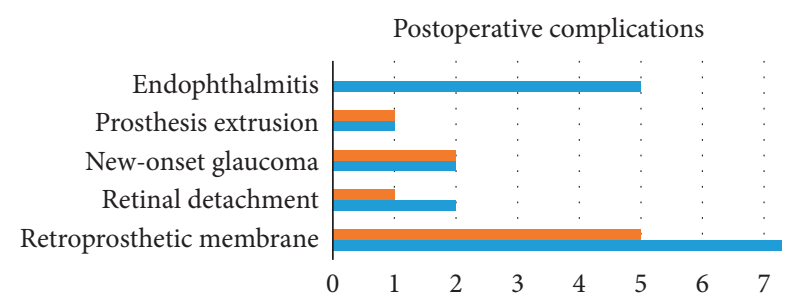

FIGURE 3: Graph illustrating the postoperative complications in the Boston type 1 keratoprosthesis (KPro) group versus the combined KPro, pars plana vitrectomy, and silicone oil insertion group.

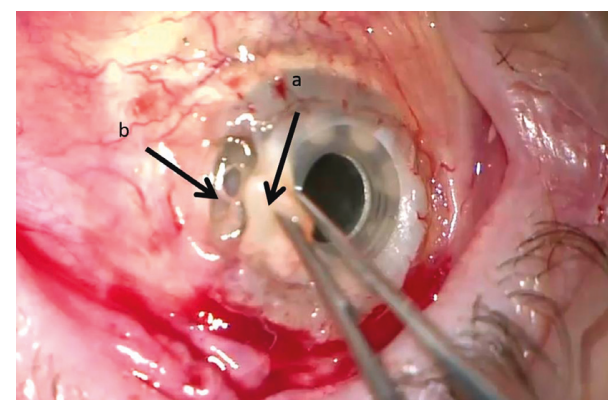

FIGURE 4: Figure depicting the melting corneal graft (a) around the Boston type 1 keratoprosthesis (KPro) of patient number \#1 in the KPro combined with the pars plana vitrectomy and silicone oil insertion group. Despite that the posterior plate of the KPro (b) is fully exposed, there is no leakage of aqueous humor or the intraocular silicone oil, secondary to the presence of a strong retroprosthetic membrane.

hypotony and retinal detachment. Silicone oil placement was combined with KPro due to a history of multiple graft failures, or a very low success rate anticipated with a traditional corneal graft, given severe hypotony and a need for chronic silicone tamponade in prephthisical eyes. Utine et al. and Chan et al. had reported on the use of KPro + PPV + SOI for the visual rehabilitation of chronic hypotony and corneal opacity $[30,31]$. Both these case series are in agreement with our study as they did not indicate that any of their patients

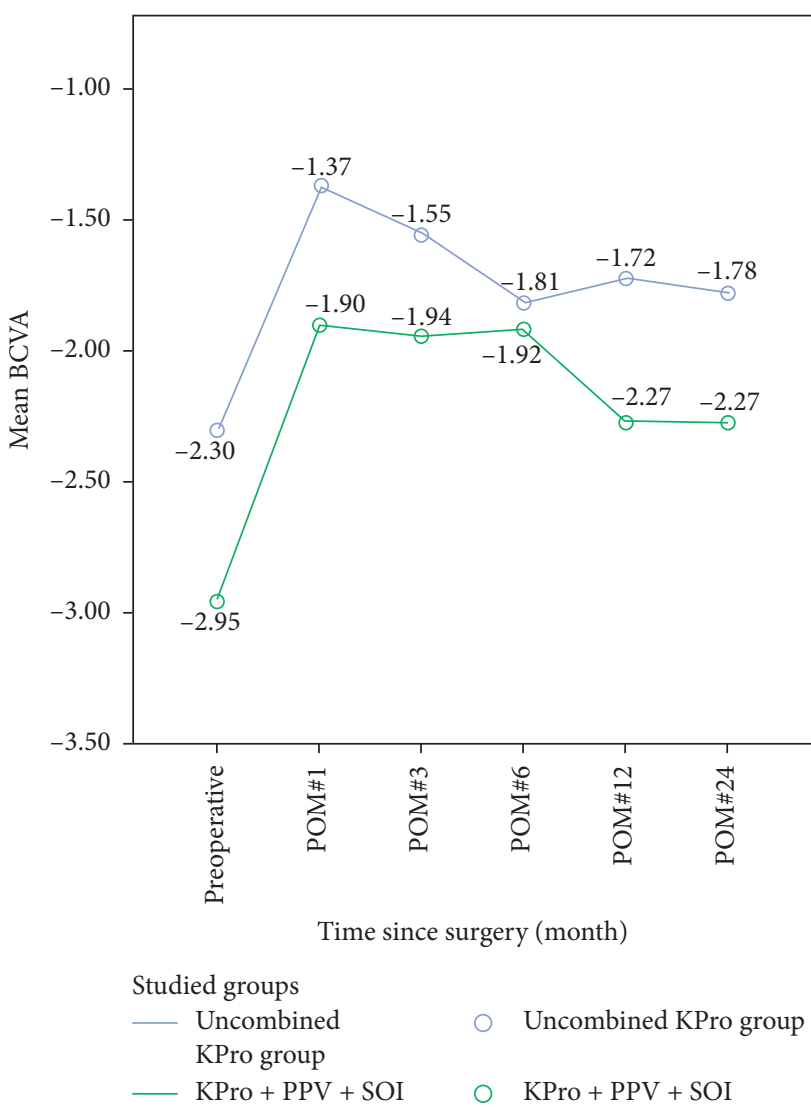

FIGURE 5: Graph illustrating the difference in the BCVA between the KPro group and the KPro + PPV + SOI group; BCVA preoperatively in the KPro group was $-2.29 \pm 0.72 \operatorname{LogMAR}$ and $-2.95 \pm 0.30 \operatorname{LogMAR}$ in the combined group $(P=0.004, t$-test $)$. After the surgery, BCVA was not statistically significant between each group.

developed endophthalmitis. Additionally, Iyer et al. reported a zero incidence of endophthalmitis in 40 silicon oil-filled eyes who underwent keratoprosthesis with a mean follow-up of 61.54 months for Boston type 1 KPro [32]. On the other hand, RPM was the most common complication (42.5\%) [32]. Our study is the first to specifically investigate the effect of combining KPro with PPV and SOI on the rate of endophthalmitis and showed that cases that underwent $\mathrm{KPro}+\mathrm{PPV}+\mathrm{SOI}$ had a significantly lower rate of endophthalmitis compared to those who underwent KPro implantation alone.

Regarding visual outcomes, there was no statistically significant difference in BCVA between both groups during the postoperative visits. This is likely related to a selection bias as those patients who received the $\mathrm{KPro}+\mathrm{PPV}+\mathrm{SOI}$ had worse visual acuity preoperatively. And as complications occurred, both groups tended to equalize. Another possible explanation for this is that silicone oil/KPro posterior plate interface induces an unanticipated high hyperopic error of refraction [33]. Further studies are needed to better describe the effect of the interface on refractive errors.

The limitations of this study include the retrospective study design, the small sample size, and the short follow-up 
period. The difference in the postoperative management was due to different preferences of the two involved surgeons. However, it is noteworthy that endophthalmitis occurred with both prophylaxis regimens. Also, we reported higher rates of endophthalmitis in the uncombined KPro group compared to the zero incidence in the combined one that could be attributed to the small sample size and the short follow-up period. A randomized prospective multicenter study with longer follow-up would be useful to further evaluate the risk to benefit ratio of the use of silicone oil in KPro patients and its role in preventing endophthalmitis and retinal complications.

In conclusion, the main outcome of our study is that PPV and SOI may have a protective effect against endophthalmitis, a vision-threatening complication not uncommon with Boston type 1 keratoprosthesis. To our knowledge, this is the first time that such effect has been demonstrated in the literature. Larger studies might further elucidate the clinical significance of this finding.

\section{Abbreviations}

KPro: $\quad$ Boston type I keratoprosthesis

KPro + PPV + SOI: Boston type 1 keratoprosthesis combined with pars plana vitrectomy and silicone oil insertion

BCVA: Best-corrected visual acuity

RPM: $\quad$ Retroprosthetic membrane

PPV: $\quad$ Pars plana vitrectomy

SOI: $\quad$ Silicone oil insertion

SLUEI: $\quad$ SAINT Louis University Eye Institute

GDI: Glaucoma drainage implant.

\section{Data Availability}

The datasets used and/or analyzed during the current study are available from the corresponding author on reasonable request.

\section{Ethical Approval}

This study was approved by the Saint Louis University and the University of Miami Institutional Review Board.

\section{Disclosure}

The funding organizations had no role in the design or conduct of this research.

\section{Conflicts of Interest}

The authors declare that there are no conflicts of interest.

\section{Authors' Contributions}

All authors analyzed and interpreted patients' data, contributed in writing the manuscript, and approved the final manuscript.

\section{Acknowledgments}

The authors thank William J Feuer, a biostatistician in Bascom Palmer Eye Institute, who helped us in the statistical analysis. This study was supported by NEI K23 award K23EY026118 (MAS), NEI core center grant to the University of Miami (P30 EY014801), and NEI R01EY018624 grant from the National Eye Institute, unrestricted grant from Research to Prevent Blindness, NY, and a grant from Alcon Research Ltd.

\section{References}

[1] A. J. Aldave, K. M. Kamal, R. C. Vo, and F. Yu, "The Boston type I keratoprosthesis: improving outcomes and expanding indications," Ophthalmology, vol. 116, no. 4, pp. 640-651, 2009.

[2] M. A. Klufas and K. A. Colby, "The Boston keratoprosthesis," International Ophthalmology Clinics, vol. 50, no. 3, pp. 161175, 2010.

[3] B. L. Zerbe, M. W. Belin, J. B. Ciolino, and Boston type 1 Keratoprosthesis Study G, "Results from the multicenter Boston type 1 keratoprosthesis study," Ophthalmology, vol. 113, no. 10, pp. e1771-e1777, 2006.

[4] D. Srikumaran, B. Munoz, A. J. Aldave et al., "Long-term outcomes of Boston type 1 keratoprosthesis implantation: a retrospective multicenter cohort," Ophthalmology, vol. 121, no. 11, pp. 2159-2164, 2014

[5] M. A. Greiner, J. Y. Li, and M. J. Mannis, "Longer-term vision outcomes and complications with the Boston type 1 keratoprosthesis at the University of California, Davis," Ophthalmology, vol. 118, no. 8, pp. 1543-1550, 2011.

[6] K. Vaziri, K. Kishor, D. Moshfeghi et al., "Incidence of blebassociated endophthalmitis in the United States," Clinical Ophthalmology, vol. 9, pp. 317-322, 2015.

[7] C. X. Zheng, M. R. Moster, M. A. Khan et al., "Infectious endophthalmitis after glaucoma drainage implant surgery: clinical features," Retina, vol. 37, no. 6, pp. 1160-1167, 2017.

[8] H. Cardona and A. G. DeVoe, "Prosthokeratoplasty," Transactions Section on Ophthalmology American Academy of Ophthalmology and Otolaryngology, vol. 83, no. 2, pp. 271280, 1977.

[9] O. Ilhan-Sarac and E. K. Akpek, "Current concepts and techniques in keratoprosthesis," Current Opinion in Ophthalmology, vol. 16, no. 4, pp. 246-250, 2005.

[10] M. Nouri, H. Terada, E. C. Alfonso, C. S. Foster, M. L. Durand, and C. H. Dohlman, "Endophthalmitis after keratoprosthesis: incidence, bacterial causes, and risk factors," Archives of Ophthalmology, vol. 119, no. 4, pp. 484-489, 2001.

[11] M. M. Nentwich, C. N. Ta, T. C. Kreutzer et al., "Incidence of postoperative endophthalmitis from 1990 to 2009 using povidone-iodine but no intracameral antibiotics at a single academic institution," Journal of Cataract \& Refractive Surgery, vol. 41, no. 1, pp. 58-66, 2015.

[12] D. G. Kent, "Endophthalmitis in Auckland 1983-1991," Australian and New Zealand Journal of Ophthalmology, vol. 21, no. 4, pp. 227-236, 1993.

[13] J. Collignon-Brach, "[Surgery for glaucoma and endophthalmitis]," Bulletin of the Belgian Societies of Ophthalmology, vol. 260, pp. 73-77, 1996.

[14] K. Mochizuki, S. Jikihara, Y. Ando, N. Hori, T. Yamamoto, and Y. Kitazawa, "Incidence of delayed onset infection after trabeculectomy with adjunctive mitomycin $\mathrm{C}$ or 5- 
fluorouracil treatment," British Journal of Ophthalmology, vol. 81, no. 10, pp. 877-883, 1997.

[15] B. Wolner, J. M. Liebmann, J. W. Sassani, R. Ritch, M. Speaker, and M. Marmor, "Late bleb-related endophthalmitis after trabeculectomy with adjunctive 5-fluorouracil," Ophthalmology, vol. 98, no. 7, pp. 1053-1060, 1991.

[16] P. W. DeBry, T. W. Perkins, G. Heatley, P. Kaufman, and L. C. Brumback, "Incidence of late-onset bleb-related complications following trabeculectomy with mitomycin," Archives of Ophthalmology, vol. 120, no. 3, pp. 297-300, 2002.

[17] D. S. Greenfield, I. J. Suñer, M. P. Miller, T. A. Kangas, P. F. Palmberg, and H. W. Flynn Jr., "Endophthalmitis after filtering surgery with mitomycin," Archives of Ophthalmology, vol. 114, no. 8, pp. 943-949, 1996.

[18] E. J. Higginbotham, R. K. Stevens, D. C. Musch et al., "Blebrelated endophthalmitis after trabeculectomy with mitomycin C," Ophthalmology, vol. 103, no. 4, pp. 650-656, 1996.

[19] M. Durand and C. Dohlman, "Successful prevention of bacterial endophthalmitis in eyes with the Boston keratoprosthesis," Cornea, vol. 28, no. 8, pp. 896-901, 2009.

[20] M. Taban, A. Behrens, R. L. Newcomb, M. Y. Nobe, and P. J. McDonnell, "Incidence of acute endophthalmitis following penetrating keratoplasty: a systematic review," Archives of ophthalmology, vol. 123, no. 5, pp. 605-609, 2005.

[21] P. Rishi, E. Rishi, V. V. Koundanya, G. Mathur, G. Iyer, and B. Srinivasan, "Vitreoretinal complications in eyes with Boston keratoprosthesis type I," Retina, vol. 36, no. 3, pp. 603-610, 2016.

[22] C. Grassi, A. Crnej, E. Paschalis, K. Colby, C. Dohlman, and J. Chodosh, "Idiopathic vitritis in the setting of Boston keratoprosthesis," Cornea, vol. 34, no. 2, pp. 165-170, 2015.

[23] S. Ray, B. F. Khan, C. H. Dohlman, and D. J. D’Amico, "Management of vitreoretinal complications in eyes with permanent keratoprosthesis," Archives of Ophthalmology, vol. 120, no. 5, pp. 559-566, 2002.

[24] R. C. Gentile, S. Shukla, M. Shah et al., "Microbiological spectrum and antibiotic sensitivity in endophthalmitis: a 25 year review," Ophthalmology, vol. 121, no. 8, pp. 1634-1642, 2014.

[25] M. S. Benz, I. U. Scott, H. W. Flynn, N. Unonius, and D. Miller, "Endophthalmitis isolates and antibiotic sensitivities: a 6-year review of culture-proven cases," American Journal of Ophthalmology, vol. 137, no. 1, pp. 38-42, 2004.

[26] F. M. Recchia, B. G. Busbee, R. B. Pearlman, C. A. CarvalhoRecchia, and A. C. Ho, "Changing trends in the microbiologic aspects of postcataract endophthalmitis," Archives of Ophthalmology, vol. 123, no. 3, pp. 341-346, 2005.

[27] D. P. Han, S. R. Wisniewski, L. A. Wilson et al., "Spectrum and susceptibilities of microbiologic isolates in the endophthalmitis vitrectomy study," American Journal of Ophthalmology, vol. 122, no. 1, pp. 1-17, 1996.

[28] A. Özdamar, C. Aras, R. Ozturk, E. Akin, M. Karacorlu, and C. Ercikan, "In vitro antimicrobial activity of silicone oil against endophthalmitis-causing agents," Retina, vol. 19, no. 2, pp. 122-126, 1999.

[29] M. P. Snead, "Ocular therapy with silicone oils," Current Opinion in Ophthalmology, vol. 4, no. 3, pp. 36-43, 1993.

[30] C. A. Utine, P. L. Gehlbach, I. Zimmer-Galler, and E. K. Akpek, "Permanent keratoprosthesis combined with pars plana vitrectomy and silicone oil injection for visual rehabilitation of chronic hypotony and corneal opacity," Cornea, vol. 29, no. 12, pp. 1401-1405, 2010.

[31] C. C. Chan, E. J. Holland, W. I. Sawyer, K. D. Neff, M. R. Petersen, and C. D. Riemann, "Boston type 1 keratoprosthesis combined with silicone oil for treatment of hypotony in prephthisical eyes," Cornea, vol. 30, no. 10, pp. 1105-1109, 2011.

[32] G. Iyer, B. Srinivasan, S. Agarwal et al., "Keratoprostheses in silicone oil-filled eyes: long-term outcomes," British Journal of Ophthalmology, vol. 103, no. 6, pp. 781-788, 2019.

[33] Z. M. A. S. M. Bodnar, R. Chod, and L. Akduman, "Hyperopic refractive error in eyes with Boston type 1 keratoprosthesis and silicone oil," Investigative Ophthalmology and Visual Science, vol. 56, no. 7, p. 1122, 2015. 


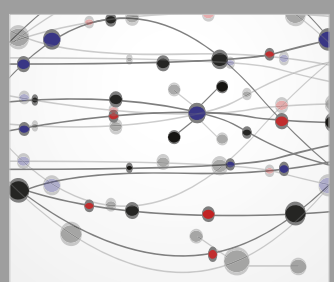

The Scientific World Journal
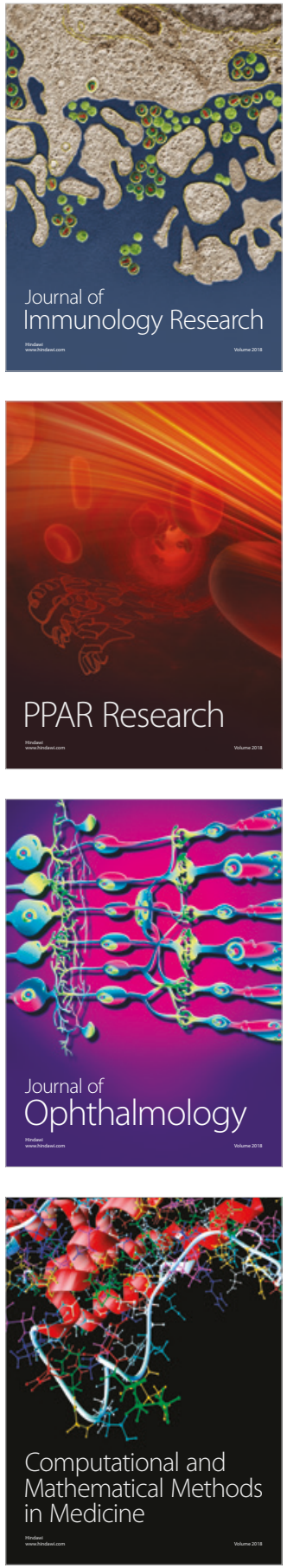

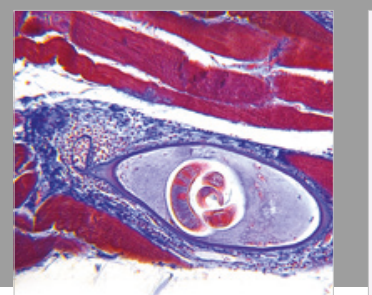

Gastroenterology Research and Practice

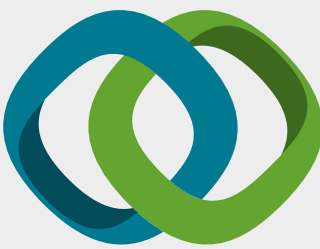

\section{Hindawi}

Submit your manuscripts at

www.hindawi.com
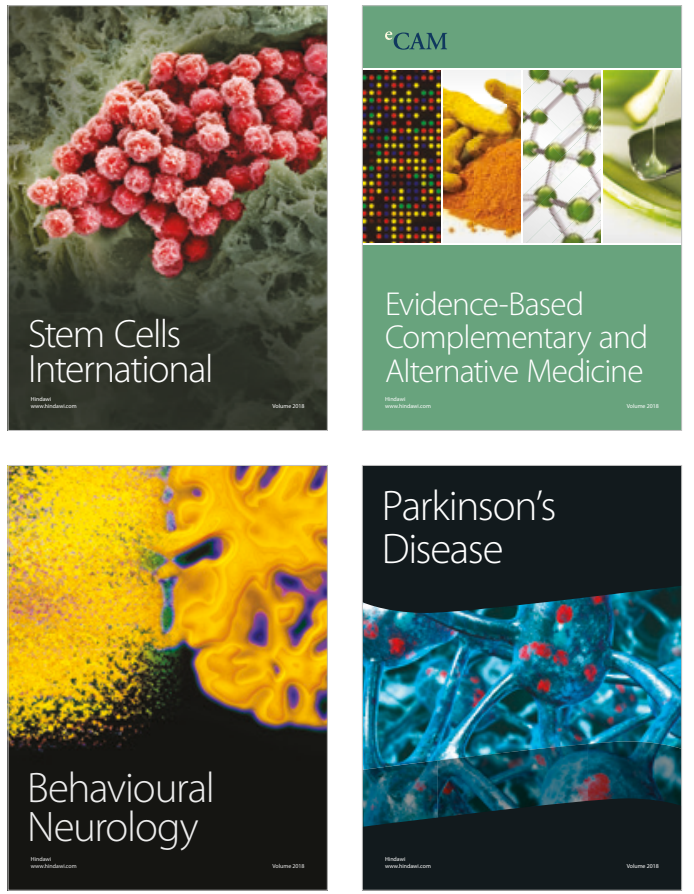

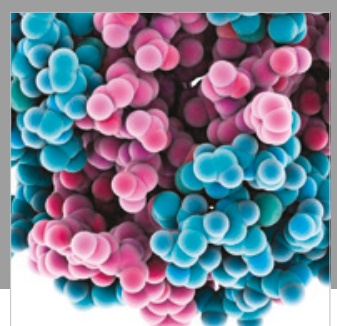

ournal of

Diabetes Research

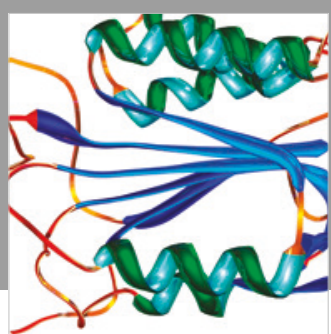

Disease Markers
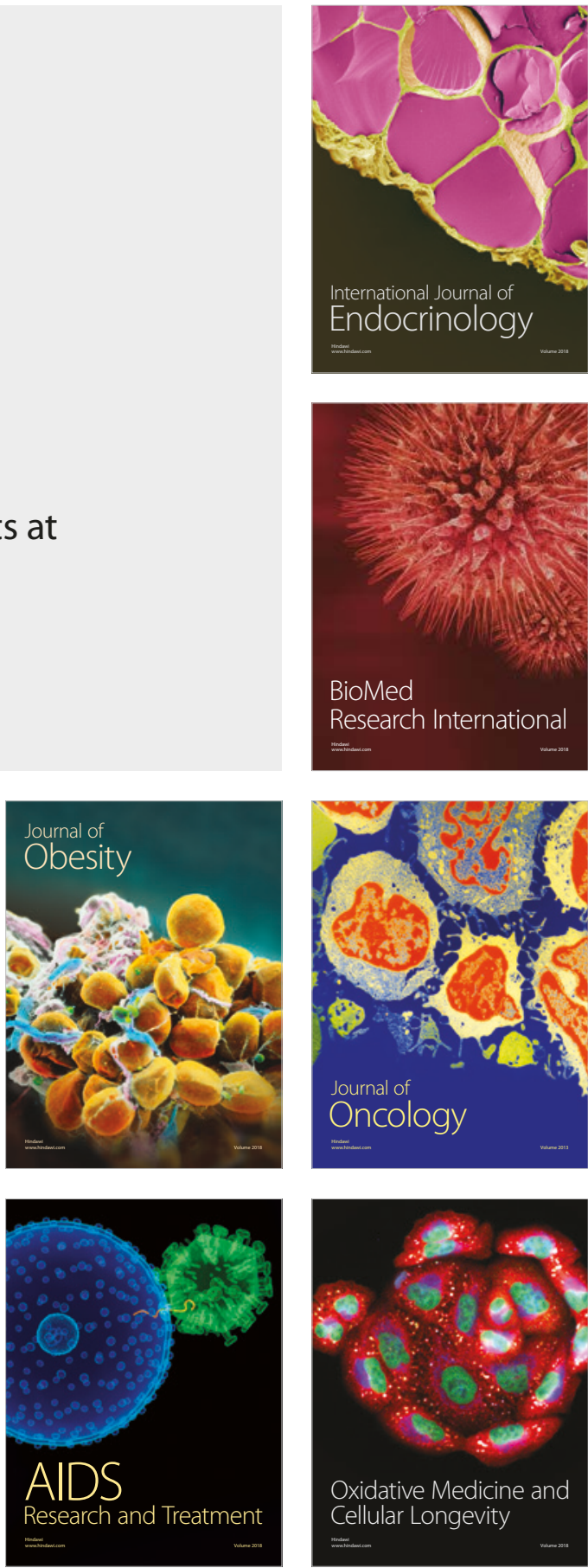